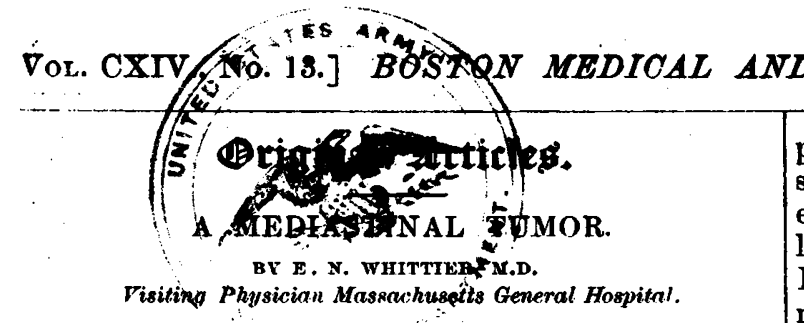

Tre condition of a patient recently under observation has suggested the topic of this paper : for the case illnstrates the distinguishing features of the disease, in the obscurity of the earlier symptoms, and the etiology ; later, in the evidences of rapid growth shown by external signs, and by local mechanical interference with the functions of the thoracic organs, and at last, in the conclusive proofs of extreme malignancy.

A man, forty-three years of age, of good family history, free from taint, without signs or history of rheumatism, alcoholism, syphilis, or Bright's disease ; by occupation a teamster, and by this subjected to much exposure and heavy exertion, had complained for two months of attacks of difficulty in breathing: attacks of such severity, and so wholly without warning or assignable cause, that he had given up work of late. Even slight changes of position in bed, from lateral to dorsal decubitus, seemed to cause dyspncea, and exertion of any kind was rendered difficult, and dreaded, by reason of suffocative attacks.

Further questioning brought out the fact of some obscure intra-thoracic disturbance eight years ago, disabling him for two months, and said by his physician to have been caused by some over-exertion in lifting; this, the patient insisted, had been fully recovered from, and never since, the source of any trouble whatever.

Paroxysms of coughing had been frequent of late, and an expectoration, at first mucous, white, and frothy, had become thinner, watery, and sometimes tinged with blood. Pain has not been a source of complaint, nor had there been palpitation nor the usual rational signs of cardiac disturbance; the voice had been unchanged, and there had been no swelling of extremities.

When first seen, three weeks previous to my later observations, the following additional facts were the only ones obtainable: At times, the face, and particularly its right side and the right side of the neck, seemed to enlarge, and he grew livid, dusky, and even purple; the veins were ready to burst, and his hands and feet, in fact, his whole body would be cold and wet, with clammy moisture, and there was great laboring for air, and constant expressions of apprehensions of impending death.

The physical signs in part corroborated his statements, and in addition, there was found swelling and slight odema above the clavicles, and increased resistance to palpation, with irregularities on deep pressure, suggesting glandular enlargements. There was less expansion above and below the left clavicle than in corresponding regions on the right side duriug inspiration, and at the junction of the third or left costal cartilage with the sternum, a limited elevation of the surface could be seen, which transmitted to the fingers a rise and fall, synchronous with the radial pulse; wellmarked dulness was found in the elevation and across the median line of the sternum, and from the left apex down to the third intercostal space; while the respiratory sounds were greatly diminished, and modified in the direction of broncho-vesicular, with some exaggeration of voice-sounds. Fine, moist râles were abundant in the remaining portion of both lings most marked posteriorly, but there was no pleural effusion on either side. The tumor communicated no thrill, nor was there evidence of lateral expansion, but the left radial was less than the right in force. The pupils were equal. Pulse 104, small, but regular; respiration 36 per minute. No abnormal condition other than these as described, were found.

There were good reasons for the two, and somewhat diverse opinions reached by the attending and consulting physicians at this date. The family physician, weighted with the obscurity of the earlier symptoms, assigning large influence to occupation, and previous history of traumatism, or over-strain, had given an opinion of aneurismal tumor. The consultant, examining without knowledge of this opinion, and without the fatigue and prejudice associated with the tedious uncertainty of the two preceding months, based his opinion largely upon the physical signs, and less upon the possible diagnostic influences of the history of the case.

If we carefully consider the symptoms which challenge careful consideration, we shall be led to regard as the most important of the symptomatic phenomena. those associated with disturbances of respiration. They are, in the order of their value in diagnosis, as follows : Rapid breathing ; dyspnoea on exertion ; dyspnœe on change of position ; dyspnoea, explained by physical signs; orthopnœa ; paroxysmal dyspnœa.

For the purposes of eliminative diagnosis, we need to consider more at length the causes of rapid breathing. They are:

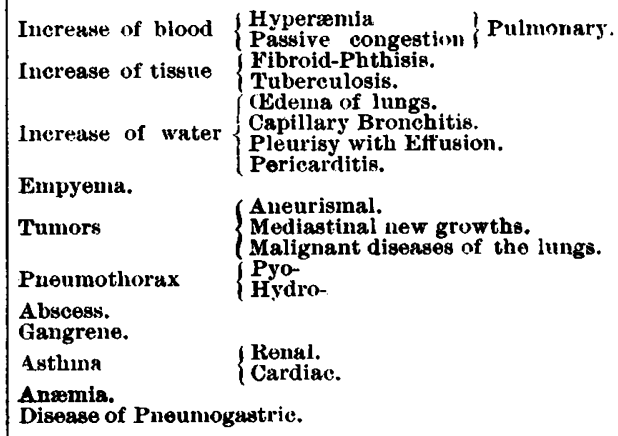

The rules of eliminative diagnosis enable us to throw out as not material to the present discussion, all of the foregoing, excepting oedema of lungs, laryngeal paralysis, and the varieties of tumors embraced under the term mediastinal new growths and aneurismal.

Pressure upon, or disease of, the pneumogastric, caused by enlarged cervical or bronchial glands, intrathoracic new growths and aneurism, is recognized as an important agent in the production of rapid breathing. The history of this case may be confidently appealed to in confirmation of the existence of one or more of the above causes.

The pulmonary cedema, joined, as it is, with evidences of venous stasis in the face and neck, cannot be wholly referable to influences exerted upon the pnenmogastric, and it is only explainable by hindrances to return of blood through the superior vena cava, by causes existing within the thoracic cavity.

I quote the admirable words of Bruen: "When we consider that in the diagnosis of aneurism of the aorta, every sigu and symptom has in turn been found to be fallacious in the ever-varying conditions under which aneurisms appear, and that one is forced to say that aneurism has no pathognomonic signs or symptoms, the

The New England Journal of Medicine. Downloaded from nejm. For personal use only. No other uses without permission. From the NEJM Archive. Copyright@ 2010 Massachusetts Medical Society. 
difficulties in the way of differential diagnosis of intrathoracic tumors may be recognized. Moreover, the diversity in the peculiarities of each case, the multifarious character of the pressure symptoms and physical signs, and the absence of a precise order of phenomena peculiar to tumors in this situation, may render a positive diagnosis in the early stages very difficult."

In this case, there had been none of the causes predisposing to disease of the coats of the arteries, but there was offsetting this, the history of laborious occupation, of violent exercise, and of obscure traumatism of seven or eight years previously; pain was nearly absent ; this, too, in spite of the evidences of a tumor, as shown by the limited prominence in the left sternal region; pulsation and thrill, murmur, and lateral expansion were absent, important diagnostic factors in aneurismal growths, and the recurrent laryngeal gave no direct sign of involvement, neither hoarseness nor aphonia having existed. The variation in the radials implied obstruction, or pressure from some one of several intra-thoracic causes, not from aneurism alone.

Mediastinal new growths, the majority of which are lympho-sarcomatous, oppose in their earlier stages many obstacles to a conclusive diagnosis. Broadly stated, there are no symptoms or physical signs, nor any precişe order of phenomena peculiar to, or definitely diagnostic of intra-thoracic new growths.

The wider the departure from the usual concurrent signs of intra-thoracic tumors; the more diffused the signs of obstruction, and of disturbed mechanism referable to the mediastinum (when thoughtfully considered in conuection with the history of the case) the firmer the foundation upon which to rest a diagnosis, of the class of intra-thoracic new growths of which one case is typical.

We were, in considering this case, mindful of the fact, that these growths increase from without inwards, that the slight external tumor might be but a surface indication of extensive deposits, an opinion confirmed by the large area of percussion dulness: that the physical signs of collapse of pulmonary lobules, upper left front, and the evidence of venous stasis in face and neck were conjoint witnesses inseparably connected and associated with the cervical glandular enlargements, an aggregation unimpeachable and conclusive.

Let us now turn to the consideration of the later symptoms found in the rapid growth of the tumor, and in the evidences of obstruction, declared by the external signs, and the local mechanical interference with the functions of the thoracic organs.

At an interval of four weeks the following conditions existed :-

The face, neck and left arm were odematous and dusky : the neck broadening to its base was much enlarged, irregular in surface, and the supra-clavicular depressions were obliterated; anteriorily, from the sternal notch upwards, the growth masked the outlines of the larynx. Respiratory movements in the left upper chest were absent; the heart's apex was displaced downwards and outwards into the seventh interspace, outside the nipple line; to rapid breathing, there had been added orthopnce, of the most pronounced type, the patient sleeping much of the time with his head bending forward towards his drawn-up knees.

The tumor before noted as a small elevation had so increased in size as to involve the costal cartilages and the ends of the second, third and fourth ribe, and measured three and one-half by four and one-half inches, with an estimated elevation of one and threefourth inches; it was a firm, resisting, non-heaving, non-expanding mass; free from pain and not tender on palpation or percussion ; there was flatness on percussion, from the left apex to the third interspace and thence to the right of the sternum. Vocal fremitus was absent in this region; respiratory murmur other than transmitted could not be heard ; the voice sounds were not distinctive ; urgent symptoms, referable to obstruction of the trachea, as well as of the superior vena cava, became more constant, and Dr. Porter's opinion was sought, with reference to surgical measures to secure relief. He decided that the growth in front of the larynx and the evidence of obstruction beneath the sternum precluded tracheotomy, and rendered any operation impracticable.

The evidences of extreme maliguancy were found in the processes destructive to life, and rapidly advancing during the few days previous to the death of the patient. Briefly these proofs were, glandular enlargements, cervical and axillary, particularly on the left side, causing obstruction and so great increase in size of the left arm that it was nearly twice the size of its fellow; cyanosis of the face and neck, taking the place of the previous lividity and dusky hue; persistent dilatation of the superficial veins of the neck and face; pressure signs referable to the trachea and left primary bronchi, and in the condition of the heart, displaced downwards and outwards, with marked signs of cardiac failure ; most significant of all, a tumor, increasing rapidly along the lines of least resistance, and involving, while not destroying, a portion of the anterior chest wall. We easily recognize in these physical signs the classical phenomena of obstruction, from a quickly developing intra-thoracic new growth.

The rational and physical signs accompanying mediastinal tumors, are complex and varying, and modified as such signs must be by reason of the changed anatomical relations of the structures involved, and the inconstant position of new growths in this region; careful consideration will, nevertheless, reveal the co-existence and close association of diagnostic factors of these three classes, namely, disorders of circulation, disturbances of innervation, respiratory phenomena.

\section{IMPERFORATE HYMEN WITH RETENTION OF THE MENSTRUAL FLUX.1}

$$
\text { BY W. H. BAKKR, M.D. }
$$

From the fact that in the practice of gynæcology for twelve years past $I$ have met with but one case, the report of which I wish to present to you this evening, assures me that as a class, they are infrequent.

We often see cases where there is more or less complete atresia of some part of the vaginal canal, as a result of a severe labor, or following some sloughing of the parts after some of the exhausting fevers; but a complete closure of the outlet of the vagina from a congenital malformation, with a well-formed vagina, uterus, and ovaries, occasioning a distention of either the vagina, uterus, or Fallopian tubes, or of all, is of much more rare occurrence.

The report of the case is as follows : Mrs. A. L. was referred to me by Dr. Towne, of Manchester, $N$. 